\title{
IS PULMONARY TUBERCULOSIS ASSOCIATED WITH SMOKELESS TOBACCO USE?
}

\author{
Amitabh Das Shukla', A. Shreenivasa², Abhinav Chaudhary3 \\ ${ }^{1}$ Associate Professor, Department of Pulmonary Medicine, Moti Lal Nehru Medical College. \\ ${ }^{2} 3^{\text {rd }}$ Year Junior Resident, Department of Pulmonary Medicine, Moti Lal Nehru Medical College. \\ $3^{33^{r d}}$ Year Junior Resident, Department of Pulmonary Medicine, Moti Lal Nehru Medical College.
}

\section{ABSTRACT}

\section{BACKGROUND}

Tobacco is one of the major causes of disease and death in India, accounting for nearly 0.9 million deaths every year. The prevalence of SLT use among adult men in India increased from 24\% in 1995 to 33\% in 2009, whereas the prevalence of smoking reduced from $35 \%$ to $24 \%$ during the same period. Several studies have demonstrated the association between smoking and the risk of pulmonary TB, relapse of TB and mortality due to TB and poor tuberculosis treatment outcome. Little data exist on the prevalence and patterns of SLT use among patients with TB in India. The objective of present study to assess the association between the tobacco chewing and pulmonary tuberculosis with respect to prevalence and mycobacterium tuberculosis bacilli load.

\section{MATERIALS AND METHODS}

A descriptive study was conducted among 257 pulmonary TB patients between January 2017 and June 2017 attending Department of Respiratory Medicine, MLN Medical College, Allahabad. Using a structured interview schedule, retrospective smoking and smokeless tobacco use data were collected at the diagnosis.

\section{RESULTS}

Total 257 pulmonary tuberculosis patients enrolled in our study, of which $178(69.2 \%)$ ever users of any form of tobacco in lifetime and 140 (54.4\%) patients were current users of any form of tobacco at the time of the survey. Among the tobacco users, 42 patients were exclusive smokers, 68 were exclusive SLT users and 30 of them used both forms of tobacco. The prevalence of tobacco chewing and smoking was $48.5 \%$ and $30 \%$ respectively at the time of diagnosis. Among tobacco chewers, the AFB smear grading was $2.9 \%$ scanty positive, $23.5 \%$ were $1+, 38.2 \%$ were $2+$ and $35.2 \%$ were $3+$ respectively.

\section{CONCLUSION}

Tobacco chewing prevalence is higher than tobacco smoking alone among patients with tuberculosis. Proper education and programmatic protocols should address against the use of tobacco products either in form of tobacco chewing or smoking which often go undetected among TB patients.

\section{KEYWORDS}

Smokeless Tuberculosis, Tuberculosis.

HOW TO CITE THIS ARTICLE: Shukla AD, Shreenivasa A, Chaudhary A. Is pulmonary tuberculosis associated with smokeless tobacco use? J. Evolution Med. Dent. Sci. 2017;6(62):4515-4517, DOI: 10.14260/Jemds/2017/976

\section{BACKGROUND}

Both tobacco chewing and tuberculosis are major global public health problems. Tobacco is one of the major causes of disease and death in India, accounting for nearly 0.9 million deaths every year. ${ }^{1}$ India is the second largest consumer and third largest producer of tobacco and tobacco products are available at very low prices. Chewing tobacco is one type of smokeless tobacco (SLT). Smokeless tobacco is available in many forms in India and is widely used by all social groups. It is more prevalent among the disadvantaged and people who live in rural areas. SLT is available in loose leaf, plug (plugfirm and plug-moist), or twist forms. The user puts a wad of tobacco inside the cheek. The estimated number of tobacco users in India was 274.9 million. Of these, 163.7 million used only SLT, 68.9 million were smokers, and 42.3 million used

Financial or Other, Competing Interest: None.

Submission 28-06-2017, Peer Review 21-07-2017,

Acceptance 27-07-2017, Published 03-08-2017.

Corresponding Author:

Dr. Amitabh Das Shukla,

Associate Professor,

Department of Pulmonary Medicine,

Moti Lal Nehru Medical College.

E-mail: adshukla1977@gmail.com

DOI: $10.14260 /$ jemds $/ 2017 / 976$ both forms of tobacco. ${ }^{2}$ The total economic costs attributed to tobacco use from all diseases in India in the year 2011 for persons aged 35-39 amounted to INR 104500 cores (US\$ 22.4 billion). ${ }^{1}$

Practically, anybody can get tuberculosis infection during a lifetime. It is potentially rapidly spread because it is airborne and spreads like the common cold, when infectious people cough, spit, talk or sneeze. The life time risk of breaking down among those infected with TB is $10-15 \%{ }^{3}$ determinants are co-infected with HIV, diabetes mellitus, smoking tobacco products, alcohol abuse, and malnutrition increases the risk progression from infection to TB disease. ${ }^{3}$ India accounts for one fourth of global TB burden i.e. 2.2 million out of 9.6 million new cases annually. ${ }^{3}$

The prevalence of SLT use among adult men in India increased from $24 \%$ in 1995 to $33 \%$ in 2009, whereas the prevalence of smoking reduced from $35 \%$ to $24 \%$ during the same period. ${ }^{2}$ Several studies have demonstrated the association between smoking and the risk of pulmonary $\mathrm{TB}, 4$ relapse of $\mathrm{TB}$ and mortality due to $\mathrm{TB}^{5}$ and poor tuberculosis treatment outcome. ${ }^{6}$ Aggressive tobacco control has been reported to avert 27 million deaths from TB attributable to smoking by $2050 . .^{7}$ There is also compelling data on the association of SLT with chronic diseases including TB.8,9 
There has been a call to implement smoking cessation strategies in TB control programmes. ${ }^{3}$ However, no such programmes were available for smokeless tobacco users. Little data exist on the prevalence and patterns of SLT use among patients with TB in India.

The objective of present study to assess the association between the tobacco chewing and pulmonary tuberculosis with respect to prevalence and mycobacterium tuberculosis bacilli load.

\section{MATERIALS AND METHODS}

\section{Design and Study Population}

A descriptive study was conducted between January 2017 and June 2017 among patients attending Department of Respiratory Medicine outpatients and inpatients clinics, MLN Medical College, Allahabad, Uttar Pradesh. To be eligible for enrolment, a patient had to be aged at least 18 years, should have provided two samples of sputum specimen that had been found smear positive for acid-fast bacilli by certified RNTCP lab where we used fluorescence staining procedure and smear grading done according to RNTCP guidelines. ${ }^{3}$

\section{Data Collection and Analysis}

At the time of study enrolment, after the patients had provided written informed consent, interview of eligible patients, most of the data were collected using a standardised questionnaire adopted from the one employed in global adult tobacco survey. ${ }^{10}$ The following data were collected: details of basic demographic and socioeconomic characteristics, disease history, pattern of past and present smokeless tobacco use and patient perceptions about links between relapse of TB and tobacco use. At the end of the study, the investigator gave tobacco cessation advice to encourage persistent and relapsed users to quit, and permanent quitters to stay abstinent from tobacco use. Analysis done by chi square test $95 \%$ confidence interval using SPSS software, $\mathrm{P}$ value $<0.05$ was considered significant.

\section{RESULTS}

A total of 1691 presumptive TB cases were enrolled in our study, out of which 257 (15.2\%, Table 1) patients having sputum smear-positive pulmonary tuberculosis, were further included in our study. The mean (SD) age of our patients was 40 (15) years (range 18-80 years). TB was more common among males $(n=179)$ than females $(n=78)$ and among 257 patients, 186 were new cases, 71 were retreatment cases who were started on Category I and category II DOTS respectively.

Of the 257 study patients, 178 (69.2\%) were ever users of any form of tobacco in lifetime out of which 140 (54.4\%) patients were current users of any form of tobacco at the time of the survey. Among the tobacco users, 42 patients were exclusive smokers, 68 were exclusive SLT users and 30 of them used both forms of tobacco [Table 2]. The prevalence of tobacco chewing and smoking was $48.5 \%$ and $30 \%$ respectively at the time of diagnosis. Among tobacco chewer, the AFB smear grading was $2.9 \%$ scanty positive, $23.5 \%$ were $1+, 38.2 \%$ were $2+$ and $35.2 \%$ were $3+$ respectively [Table 3 ].

\begin{tabular}{|c|c|c|}
\hline $\begin{array}{c}\text { Sputum AFB Smear } \\
\text { Status }\end{array}$ & $\begin{array}{c}\text { Tobacco } \\
\text { User }\end{array}$ & $\begin{array}{c}\text { Non Tobacco } \\
\text { User }\end{array}$ \\
\hline Positive & 178 & 79 \\
\hline Negative & 286 & 1159 \\
\hline Table 1. Prevalence of Tuberculosis and Tobacco use in \\
Presumptive Tuberculosis Case \\
\hline
\end{tabular}

\begin{tabular}{|c|c|c|}
\hline Type & Ever use (\%) & Current use (\%) \\
\hline Any form of tobacco & $178(69.2)$ & $140(54.4 \%)$ \\
\hline Tobacco chewing only & $28(10.8)$ & $68(26.4)$ \\
\hline Tobacco smoking only & $57(22.2)$ & $42(16.3)$ \\
\hline Both form of use & $93(36.1)$ & $30(11.6)$ \\
\hline $\begin{array}{c}\text { Never used any form of } \\
\text { tobacco }\end{array}$ & $79(30.8)$ & $117(45.6)$ \\
\hline
\end{tabular}

Table 2. Patterns of Tobacco use among Pulmonary Tuberculosis Patients. ( $\mathrm{n}=257)$

At the time of diagnosis.

\begin{tabular}{|c|c|c|}
\hline $\begin{array}{c}\text { Sputum AFB } \\
\text { Smear Grading }\end{array}$ & $\begin{array}{c}\text { Tobacco Chewer } \\
(\mathbf{\%})(\mathbf{n}=\mathbf{6 8})\end{array}$ & $\begin{array}{c}\text { Non Tobacco User } \\
(\mathbf{n}=\mathbf{6 8})\end{array}$ \\
\hline Scanty & $2(2.9)$ & $6(8.8)$ \\
\hline $1+$ & $16(23.5)$ & $30(44.1)$ \\
\hline $2+$ & $26(38.2)$ & $18(26.5)$ \\
\hline $3+$ & $24(35.2)$ & $14(20.5)$ \\
\hline Table 3. Comparison of Sputum AFB Smear Grading \\
among Smokeless Tobacco Users and Non-Tobacco Users \\
\hline
\end{tabular}

$P$ value $=0.015$

\section{DISCUSSION}

In our study, $69.2 \%$ of 257 patients with pulmonary tuberculosis surveyed in Department of Pulmonary Medicine, MLN Medical College, Allahabad, ever used some form of tobacco. The prevalence of current use of tobacco chewing in our study is $26.4 \%$ as compared to the prevalence of SLT among newly diagnosed patients with TB from Malaysia $(29 \%)^{11}$ and $29.7 \%$ from study conducted by K.G. Deepak et $\mathrm{al}^{9}$ in Udupi district, Karnataka. The prevalence of ever smoking only among TB patients was $22.2 \%$, which was lower than that reported from K.G. Deepak et al $^{9}$ and $27.9 \%$ among adult men in Mumbai Karnataka. ${ }^{12}$

From this study we found that $26.4 \%$ sputum-positive TB patients were addicted to tobacco chewing, $16.3 \%$ patients were addicted to smoking and $11.6 \%$ patients were users of both forms of tobacco which is almost similar to data reported by K.G. Deepak et al. ${ }^{9}$ A South African study observed that $56 \%$ of patients with active tuberculosis were current smokers. ${ }^{13}$ Similarly, $54.6 \%$ of Chinese patients with tuberculosis were smokers ${ }^{14}$ and $59.9 \%$ of the Georgia tuberculosis patients were either current smokers or individuals who had ceased smoking no more than two months earlier. ${ }^{6}$ In our study, we found that sputum bacillary load which was graded according to RNTCP guidelines $^{3}$ was higher among tobacco users as compared to non-tobacco user TB patients.

The use of smokeless tobacco products (Tobacco chewing) was much higher among active TB patients than that of smoking. Smokeless tobacco products are linked to oral cancers in India ${ }^{15}$ and studies have also shown that the use of SLT increases the risk of death from respiratory diseases and TB in men. A perception that smokeless tobacco use is less harmful for patients with TB needs to be corrected, because of the serious health consequences of chewing 
tobacco. Most of the tobacco messages provided by doctors to patients are used to be general in nature and focused on smoking. Hence, these patients were advised to give up smoking and alternatively were engaged in the use of SLT as a form of harm reduction, and as a nicotine substitution strategy. This study suggests that more tobacco and TB specific cessation messages need to be given to these patients.

There has been a recent call to include tobacco cessation as a routine part of TB management protocols. ${ }^{3}$ In India, where the chewing as well as the smoking form of tobacco is common, tobacco cessation efforts must focus on both smoking and tobacco chewing. We also found that many patients with TB or patients with respiratory symptoms prefer not to smoke, but continue tobacco chewing after diagnosis. Healthcare worker's effort to advise TB patients to quit tobacco during and following treatment needs to be encouraged as a routine part of TB counselling and support. Likewise, DOTS providers and healthcare professionals need to be trained to offer structured tobacco cessation advice to patients and offer support.

Our study had several limitations. First, our primary exposure variable - self-reported tobacco addiction status was subject to potential misclassification. Data on tobacco use behaviour relies on self-report and may suffer from recall bias.

\section{CONCLUSION}

Tobacco chewing prevalence is higher than tobacco smoking alone among patients with tuberculosis. Proper education and programmatic protocols should address against the use of tobacco products either in form of tobacco chewing or smoking which often go unheeded among TB patients. Patients with tuberculosis are advised by their doctors, at the time of diagnosis, to quit smoking. Several patients shift from smoking to smokeless tobacco use, which needs to be addressed while providing tobacco cessation services. Proactive efforts are needed to encourage health care staff and DOTS providers to give strong cessation messages.

\section{REFERENCES}

[1] World

Health Organization http://www.searo.who.int/india/topics/tobacco/en/.

[2] International institute for population sciences (IIPS) and ministry of health and family welfare (MoHFW). Global adult tobacco survey (GATS) India 2009-2010. Mumbai and New Delhi: IIPS and MoHFW, government of India 2010.

[3] Central TB division, Director general of health service. TB India 2016: RNTCP technical and operational guidelines for tuberculosis control in India 2016. Ministry of health and family welfare, government of India./www.tbcindia.gov.in 2016.
[4] Kolappan C, Gopi PG. Tobacco smoking and pulmonary tuberculosis. Thorax 2002;57(11):964-6.

[5] Chiang CY, Slama K, Enarson DA. Associations between tobacco and tuberculosis. Int J Tuberc Lung Dis 2007;11(3):258-62.

[6] Gegia M, Magee MJ, Kempker RR, et al. Tobacco smoking and tuberculosis treatment outcomes: a prospective cohort study in Georgia. Bulletin of the World Health Organisation 2015;93(6):390-9.

[7] Basu S, Stuckler D, Bitton A, et al. Projected effects of tobacco smoking on worldwide tuberculosis control: mathematical modelling analysis. BMJ 2011;343:d5506.

[8] Gupta PC, Pednekar MS, Parkin DM, et al. Tobacco associated mortality in Mumbai (Bombay) India. Results of the Bombay Cohort Study. Int J Epidemiol 2005;34(6):1395-402.

[9] Deepak KG, Daivadanam M, Pradeepkumar AS, et al. Smokeless tobacco use among patients with tuberculosis in Karnataka: the need for cessation services. The National Medical Journal of India 2012;25(3):143-5.

[10] Global adult tobacco survey collaborative group. Tobacco questions for surveys: a subset of key questions from the global adult tobacco survey. $2^{\text {nd }}$ edn. Atlanta: centers for disease control and prevention 2011.

[11] Awaisu A, Mohamed NMH, Aziz AN, et al. Tobacco use prevalence, knowledge, and attitudes among newly diagnosed tuberculosis patients in Penang state and Wilayah Persekutuan Kuala Lumpur, Malaysia. Tob Induc Dis 2010;8(1):3.

[12] International institute for population sciences (IIPS) and macro international. National family health survey (NFHS-3), 2005-06, India: Key findings. Mumbai: IIPS 2007.

[13] Brunet L, Pai M, Davids V, et al. High prevalence of smoking among patients with suspected tuberculosis in South Africa. Eur Respir J 2011;38(1):139-46.

[14] Wang J, Shen H. Review of cigarette smoking and tuberculosis in China: intervention is needed for smoking cessation among tuberculosis patients. BMC Public Health 2009;9(1):292.

[15] Muwonge R, Ramadas K, Sankila R, et al. Role of tobacco smoking, chewing and alcohol drinking in the risk of oral cancer in Trivandrum, India: a nested case-control design using incident cancer cases. Oral Oncol 2008;44(5):446-54. 